\title{
Geraniol attenuates osteoclast differentiation by suppressing NF-kB activity and expression of osteoclastogenic genes
}

\author{
Vishwa Deepak ${ }^{1}$, Marlena C Kruger ${ }^{2,3,4}$, Magdalena Coetzee ${ }^{1,4, \varpi}$ \\ ${ }^{\square}$ Dr. Magdalena Coetzee \\ E-Mail: magdalena.coetzee@up.ac.za \\ ${ }^{1}$ Department of Physiology, \\ University of Pretoria, \\ Pretoria, South Africa \\ ${ }^{2}$ School of Food and Nutrition, \\ Massey Institute of Food Science and Technology, \\ Massey University, Palmerston North, New Zealand \\ ${ }^{3}$ Department of Human Nutrition, \\ Faculty of Health Sciences, \\ University of Pretoria, \\ Pretoria, South Africa \\ ${ }^{4}$ Associate of the Institute for Food, \\ Nutrition and Well-being, \\ University of Pretoria, \\ Pretoria, South Africa
}




\begin{abstract}
Osteoporotic patients have lower bone mass due to increased bone resorption by osteoclasts. The aim of this study was to investigate the cytotoxic and anti-osteoclastogenic effects of geraniol, a natural monoterpene on human CD14+ monocytes (ex vivo) and murine RAW264.7 macrophages (in vitro) using alamar blue and tartrate resistant acid phosphatase (TRAP) staining respectively. The anti-osteoclastogenic activity of geraniol was further explored by analyzing its effects on actin ring formation and bone resorptive function of osteoclasts. Geraniol significantly $(p<0.001)$ inhibited osteoclast formation in CD14+ monocytes and RAW264.7 macrophages without cytotoxicity. Moreover, reduced osteoclastogenesis in these cells led to an arrest in actin ring formation and diminished bone resorption. Analysis of underlying molecular mechanisms revealed that geraniol alleviated NF-kB activity, an indispensable upstream modulator of osteoclast formation. Furthermore, expression of key osteoclastogenic genes such as dendritic cell-specific transmembrane protein (DC-STAMP) involved in cell-cell fusion and nuclear factor of activated T-cells, cytoplasmic, calcineurin-dependent 1 (NFATc1), a master transcription factor essential for osteoclast differentiation was downregulated by geraniol. These observations indicate that inhibition of osteoclast differentiation is presumably one of the pharmacological properties of geraniol.
\end{abstract}

Keywords: Osteoporosis; Bone; RANKL; Osteoclast differentiation; DC-STAMP; NFATc1 


\section{Introduction}

Low bone mass and weakened bone tissue microarchitecture is one of the features of osteoporosis (Drake et al., 2015). Compromised bone architecture not only increases the risk of fractures but also augments mortality risks. Bone remodeling occurs in an orchestrated manner where osteoblastic bone formation proceeds osteoclastic bone resorption during the lifetime of an individual (Hadjidakis and Androulakis 2006). Repair and maintenance of bone tissue homeostasis is critical for a healthy skeleton. An abrupt and unregulated increase in bone resorption disturbs the balance in bone remodeling often seen during several pathological bone diseases such as osteoporosis, periodontitis, arthritis and cancer (Mundy 1999).

Osteoclasts are the sole bone-resorbing multinucleated cells of the body arising from the fusion of hematopoietic cells belonging to the monocyte/macrophage lineage (Boyle et al., 2003). Receptor activator of NF-kB ligand (RANKL) is the key cytokine that regulates differentiation of boneresorbing osteoclasts (Soysa and Alles 2009). Binding of RANKL to RANK on the cell surface of osteoclast precursors triggers downstream signaling events that lead to the induction of osteoclastogenesis (Wada et al., 2006). Several studies have confirmed the critical role of the NF$\mathrm{kB}$ signaling in the differentiation, function, and survival of osteoclasts in vitro and in vivo (Vaira and Johnson and et al., 2008, Vaira and Alhawagri and et al., 2008, Iotsova et al., 1997). NF-kB activation is a key signaling event triggered by the osteoclastogenic cytokine RANKL to stimulate osteoclast formation (Soysa and Alles 2009). Accumulating lines of evidence suggest that abrupt activation of NF-kB pathway further contributes to pathological osteoclastogenesis, and pharmacological inhibition of NF-kB retards in vivo osteoclastogenesis and ovariectomy-induced bone loss (Strait et al., 2008). Antagonists of NF-kB have been shown to prevent bone loss 
associated with estrogen deficiency in mice (Strait et al., 2008), in animal models of rheumatoid arthritis (Dai et al., 2004) and in bone loss associated with cancer (Feng et al., 2007).

Multinucleated osteoclasts express tartrate resistant acid phosphatase (TRAP) (Hayman 2008) and attach to the bone matrix where they secrete lytic enzymes such as cathepsin K (CTSK) into the resorption lacuna surrounded by a tight sealing zone (Boyle et al., 2003). The sealing zone is a hallmark feature of a functional osteoclast that segregates the resorptive microenvironment from the general extracellular space. Osteoclast specific lysosomal proteases play an important role in bone homeostasis, including the solubilization of bone matrix (Delaisse et al., 2003). Cysteine proteases such as CTSK has been identified as a crucial protease involved in bone matrix degradation (Ortega et al., 2003, Costa et al., 2011). Earlier published reports show that dendritic cell-specific transmembrane protein (DC-STAMP) is a RANKL-induced protein that is indispensable for multinucleated osteoclast formation (Yagi et al., 2005).

Natural products have widely been used as a novel source of small molecules for drug discovery. Several of the currently FDA approved drugs are either derived or based on them (Dias et al., 2012). Recent reports suggest that various naturally occurring small molecules have bone protective effects both in vitro and in vivo. For instance, compounds such as daidzein (Fonseca and Ward 2004), eugenol (Deepak and Kasonga and et al., 2015), piperine (Deepak and Kruger and et al., 2015) and $\beta$-Cryptoxanthin (Uchiyama and Yamaguchi 2005) have been reported to prevent bone loss, and reduce osteoclastogenesis in vitro. Terpenes are a class of natural products abundantly present in medicinal plants and confer several pharmacological properties (Singh and Sharma 2015). Geraniol found in rose oil, palmarosa oil, and citronella oil is an acyclic monoterpene approved by the Food and Drug Administration (FDA) for use as a synthetic flavouring and adjuvant for direct addition to food for human consumption (Chen and Viljoen 
2010). Geraniol possesses anti-oxidative, anti-microbial (Tsai et al., 2011) and anti-inflammatory activities (Medicherla et al., 2015). Furthermore, it suppresses tumor growth by targeting cell cycle and apoptosis pathways (Kim et al., 2011). However, to date the direct effects of geraniol on bone lytic cells have not been studied. In this study, we explored the anti-osteoclastogenic effects of geraniol and its underlying molecular mechanisms.

\section{Material and methods}

\section{Materials}

Geraniol and all other reagents of research grade (unless mentioned otherwise) were obtained from Sigma Aldrich Inc. (St Louis, MO).

\section{Cell culture of murine RAW264.7 macrophages}

RAW264.7 murine macrophages (\#TIB-71) were purchased from American Type Culture Collection (ATCC, Rockville, MD) and maintained in DMEM with 10\% FBS as described previously (Visagie et al., 2015).

\section{Isolation and cell culture of human CD14+ monocytes}

Peripheral blood (40-60 ml) of healthy male donors (aged 18-35) was used to isolate monocytes by centrifugation on a Ficoll-Paque gradient and magnetically sorted using a CD14+ magnetic beads isolation kit as per manufacturer's instructions (Miltenyi Biotec, CA). Cells were cultured in $\alpha$-MEM supplemented with $10 \%$ FBS and incubated at $37^{\circ} \mathrm{C}$ in a humidified atmosphere with 7\% $\mathrm{CO}_{2}$ as described previously (Deepak, Kruger et al., 2015). 


\begin{abstract}
Alamar blue assay
Cells $\left(5 \times 10^{3}\right)$ were seeded in 96 -well plates and allowed to adhere for $12 \mathrm{~h}$ followed by exposure to increasing concentrations of geraniol $(7 \mu \mathrm{g}-23 \mu \mathrm{g} / \mathrm{ml})$ for $48 \mathrm{~h}$. Alamar blue assay was conducted as per manufacturer's instructions (Life Technologies, CA). Absorbance was measured at $570 \mathrm{~nm}$ with $600 \mathrm{~nm}$ as reference wavelength on a microplate reader (BioTek Instruments Inc., VT).
\end{abstract}

\title{
Osteoclast differentiation and TRAP staining
}

RAW264.7 macrophages were differentiated into osteoclasts as previously described (Deepak, Kasonga et al., 2015). Briefly $5 \times 10^{3}$ cells per well of a 96-well plate were stimulated with RANKL (R\&D Systems, MN) alone (15 ng/ml) or in combination with increasing concentrations of geraniol $(0 \mu \mathrm{g}-23 \mu \mathrm{g} / \mathrm{ml})$. Cell culture media and factors were replaced every third day and differentiation was terminated on the fifth day unless otherwise stated.

CD14+ monocytes $\left(5 \times 10^{4}\right)$ were differentiated in the presence of M-CSF $(25 \mathrm{ng} / \mathrm{ml}, \mathrm{R} \& \mathrm{D}$ Systems, MN) and RANKL (30 ng/ml, Insight Biotechnology, Middlesex, UK) in 96 well plates for 14 days as described previously (Deepak, Kruger et al., 2015).

Osteoclast specific TRAP staining was performed using a leucocyte acid-phosphatase kit as per manufacturer's directions (Sigma Aldrich, St Louis). TRAP+ cells with 3 or more nuclei were scored as osteoclasts. Photomicrographs were taken with a Zeiss Axiocam MRc5 camera attached to a Zeiss Axiovert 40 CFL microscope (Carl Zeiss AG, Oberkochen, Germany). 


\section{Resorption pit formation assay}

The bone resorption activity of RANKL-induced osteoclasts derived from RAW264.7 macrophages was assessed using osteoassay plates as per manufacturer's instructions (Corning Inc, New York). Briefly, RAW264.7 macrophages $\left(1.5 \times 10^{4}\right.$ cells/well $)$ were seeded onto Corning Osteo Assay 24-well plates. Cells were either seeded in the presence of $15 \mathrm{ng} / \mathrm{ml}$ RANKL alone or in combination with geraniol $(23 \mu \mathrm{g} / \mathrm{ml})$. Media and factors were replaced on days 3 and 6 . After 8 days, cells were washed off the bottom of the plates with a 5\% bleach solution. Resorbed areas on the plates were captured with a Zeiss Axiocam ERc5 microscope attached to a Zeiss Axiocam MRc5 camera. Resorption pits were quantified by ImageJ software.

\section{Actin ring formation assay}

Actin rings of osteoclasts were detected by staining actin filaments with Atto-conjugated phalloidin as described previously (Visagie et al., 2015). Briefly, RAW264.7 macrophages (5× $10^{3}$ cells/well) were seeded onto 96-well plates. After overnight attachment cells were either treated with $15 \mathrm{ng} / \mathrm{ml}$ RANKL alone or in combination with geraniol $(23 \mu \mathrm{g} / \mathrm{ml})$. Cells were differentiated for 5 days with a change of medium and factors after every 3 days. Cells were washed with PBS and fixed with a $3.7 \%$ formaldehyde solution. Cells were permeabilized with $0.2 \%$ Triton X-100 in PBS for 10 min and were stained with fluorescent phalloidin conjugate solution for $40 \mathrm{~min}$ at room temperature. Actin rings were visualised using a Zeiss inverted Axiovert CFL40 microscope attached to a Zeiss Axiovert camera.

\section{NF-kB Secreted Embryonic Alkaline Phosphatase - Promoter (SEAP) assay}

pNiFty2-SEAP (Invivogen, CA) is an NF- $\kappa B$-inducible reporter plasmid containing 5x NF-kB repeated transcription factor binding sites and a reporter gene - SEAP. RAW264.7 macrophages were stably transfected with GeneCellin ${ }^{\mathrm{TM}}$ transfection reagent. Stably transfected clones 
containing SEAP plasmid were selected with Zeocin. For promoter assay the transfected cell line was stimulated with $35 \mathrm{ng}$ of RANKL in the presence or absence of geraniol and SEAP assay was conducted after $48 \mathrm{~h}$ as per manufacturer's protocol (Invivogen, CA). Briefly, following the incubation period, $10 \mu 1$ of cell culture supernatant was transferred to a 96-well plate and incubated at $65^{\circ} \mathrm{C}$ for $5 \mathrm{~min}$ to inhibit endogenous alkaline phosphatase. Fifty $\mu$ l of $1 \mathrm{X}$ Dilution Buffer, 100 $\mu 1$ of $1 \mathrm{X}$ Assay Buffer, $20 \mu \mathrm{l}$ of $100 \mathrm{mM} \mathrm{L-Homoarginine} \mathrm{and} 20 \mu 1 \mathrm{H}_{2} \mathrm{O}$ was added to each well and further incubated at $37^{\circ} \mathrm{C}$ for $10 \mathrm{~min}$. Staining Solution $(20 \mu \mathrm{l})$ was added to each well and subsequently incubated at $37^{\circ} \mathrm{C}$ for $30 \mathrm{~min}$ followed by reading the plate at OD $405 \mathrm{~nm}$. Readings from the control (without RANKL and inhibitor) were subtracted from each well to nullify the background activity.

\section{RNA extraction and Real time RT-PCR}

Total RNA was isolated from cells using the TRI reagent and reverse transcribed into cDNA using M-MuLV Reverse Transcriptase (New England Biolabs, MA). Real-time PCR was performed to analyze the differential expression of selected osteoclastogenic genes using a Roche LightCycler Nano system (Roche Diagnostics, Switzerland) and SYBR Fast qPCR Master Mix (Kapa Biosystems, South Africa). The following primer sets were used: CTSK: (forward) 5'CTGGAGGGCCAACTCAAGA-3'， (reverse) 5'-CCTCTGCATTTAGCTGCCTT-3'; TRAP: (forward) 5'-CCACCCTGAGATTTGTGGCT-3', $\quad$ (reverse) 5'ACATACCAGGGGATGTTGCG-3'; $\quad$ DC-STAMP: $\quad$ (forward) 5'- $^{\prime}$ ATGACTTGCAACCTAAGGGCAAAG-3', (reverse) 5'GTCTGGTTCCAAGAAACAAGGTCAT-3'; NFATC1 (forward) 5'GTGGAGAAGCAGAGCAC-3'， (reverse) 5'-ACGCTGGTACTGGCTTC-3’; GAPDH (forward) 5'-GATGACATCAAGAAGGTGGTGAAGC-3', $\quad$ (reverse) 5'- 
ATACCAGGAAATGAGCTTGACAAAG-3'. The values obtained from each sample were normalized to GAPDH (Glyceraldehyde-3-Phosphate Dehydrogenase) expression. The foldchange was calculated using $2^{-\Delta \Delta \mathrm{CT}}$ method.

\section{Statistical analysis}

Data are representative of three independent experiments and are represented as mean \pm standard deviation (SD). Statistical analysis was performed with one-way analysis of variance (ANOVA) followed by Bonferroni post hoc test using Graph Pad Prism Software (GraphPad Software Inc., CA). P <0.05 was regarded as statistically significant.

\section{Results}

\section{Effects of geraniol on the viability of CD14+ monocytes and RAW264.7 macrophages}

CD14+ monocytes and RAW264.7 macrophages were exposed to geraniol (7 $\mu \mathrm{g}-23 \mu \mathrm{g} / \mathrm{ml})$ to evaluate cell viability by alamar blue assay. Geraniol at the tested concentrations did not exert cytotoxic effects on the studied cell lines (Fig. 1 A, B), and hence downstream experiments were conducted amongst these concentrations.

\section{Geraniol inhibits osteoclast differentiation in CD14+ monocytes and RAW264.7}

\section{macrophages}

We employed human CD14+ monocytes as an ex vivo model and murine RAW264.7 macrophages as an in vitro model to study the effects of geraniol on osteoclast differentiation. Both the cell lines experienced a remarkable increase in TRAP+ multinucleated osteoclast formation in the presence of RANKL (Fig 2 A-D). In contrast, geraniol dose-dependently blunted the differentiation of the progenitors into osteoclasts (Fig 2 A-D). 


\section{Geraniol inhibits actin ring formation and bone resorption}

Cytoskeletal rearrangement and ruffled border formation is essential for osteoclasts to resorb bone. To investigate the inhibitory effects of geraniol on actin ring formation and bone resorption, we analyzed the effects of this compound by phalloidin staining and bone resorption assay on osteoassay plates. RANKL induced formation of clear and distinct actin rings in osteoclasts differentiated from RAW264.7 macrophages at the fifth day of differentiation (Fig 3A). In contrast, cells treated with geraniol $(23 \mu \mathrm{g} / \mathrm{ml})$ failed to fuse into multinucleated osteoclasts and thereby experienced an arrest in actin ring formation (Fig 3A). Concomitantly, absence of actin rings in geraniol-treated cells led to a complete abrogation in the resorptive potential of these cells when compared to RANKL-treated cells, showing more than $90 \%$ resorption of the surface area (Fig 3B).

\section{Geraniol suppresses RANKL-induced NF-kB activation}

NF-kB pathway is a critical mediator of osteoclast differentiation. To investigate the antiosteoclastogenic effects of geraniol, we conducted a NF-kB-secreted-alkaline phosphatasepromoter reporter assay (SEAP). pNiFty2-SEAP plasmid contains 5x-NF-kB repeated transcription factor binding sites. RAW264.7 macrophages stably transfected with the SEAP plasmid were co-treated either with RANKL or in the absence and presence of geraniol for $48 \mathrm{~h}$. Intriguingly, geraniol significantly suppressed RANKL-induced NF-kB promoter activity at 15 and $23 \mu \mathrm{g} / \mathrm{ml}$ (Fig. 4).

Geraniol reduces the mRNA expression of genes involved in osteoclast fusion and bone resorption 
Binding of RANKL to its receptor RANK triggers the expression of genes critical for osteoclast formation and function. The induction of NFATc1 in osteoclast precursors is essential for osteoclast differentiation. DC-STAMP is crucial for the fusion of osteoclast progenitors whereas TRAP expression is regarded as an important marker of osteoclasts. CTSK is a lysosomal protease responsible for bone resorption mediated by mature osteoclasts. RANKL treatment upregulated the expression of CTSK (14 fold), TRAP (>50 fold), NFATc1 ( 8 fold) and DC-STAMP (12 fold) (Fig. 5). On the contrary, co-exposure of these cells with geraniol $(23 \mu \mathrm{g} / \mathrm{ml})$ led to a significant downregulation in the expression of these genes (Fig. 5).

\section{Discussion}

Bone formation and resorption is constantly maintained by the coupled action of osteoblasts and osteoclasts within the bone milieu (Hadjidakis and Androulakis 2006). This dynamic interplay of formation and resorption is often abrogated during diseases such as chronic inflammation, osteoporosis, periodontitis, arthritis and cancer (Mundy 1999). Unwarranted increase in osteoclast formation leads to heightened bone erosion rather than reduced osteoblast activity. Consequently, drugs that target osteoclast formation can prevent osteolytic diseases. The RANKL signaling pathway is crucial for osteoclast formation and is an important druggable target for developing therapeutics against pathological diseases of bone loss (Cote 2015, Tat et al., 2009, McGrath 2011). In this study, we found that geraniol significantly alleviated the RANKL-induced osteoclast differentiation of RAW264.7 macrophages and CD14+ monocytes. Our results suggest that geraniol-mediated anti-osteoclastogenic activity might be due to downregulation of NF-kB activation, and synergistic reduction in the expression of NFATc1 and other key osteoclastogenesis genes. 
Natural products possess potent pharmacological properties and have led to development of many drugs (Dias et al., 2012). In search of novel anti-osteoclastogenic compounds we evaluated the efficacy of geraniol on osteoclast formation. We found that geraniol potently mitigated RANKLinduced TRAP+ osteoclast formation without exerting cytotoxic effects. Osteoclast formation involves fusion of progenitor cells and cytoskeleton rearrangement resulting in actin ring formation. These rings aid osteoclasts in attachment, sealing zone formation and resorption of bone (Boyle et al., 2003). We found that abrogated osteoclastogenesis in geraniol-treated cells led to failure in actin ring formation and inhibition of resorption pit formation.

NF-kB pathway plays a vital role in osteoclastogenesis (Soysa and Alles 2009). NF-kB knockout mice display severe osteopetrosis due to complete lack of osteoclasts (Iotsova et al., 1997). During osteoclastogenesis the NF-kB pathway is activated leading to downstream signaling events and osteoclast-specific gene expression (Strait et al., 2008). Hence, targeting NF-kB activation could also lead to disruption in osteoclast formation and bone loss. It has been reported that several compounds targeting this pathway have bone protective properties and are capable of anabolic effects, including the estrogen, 17b-estradiol (Yamaguchi and Weitzmann 2009), piperine (Deepak, Kruger et al., 2015) and eugenol (Deepak, Kasonga et al., 2015). Our SEAP promoter assay results demonstrate that NF-kB was activated by RANKL whereas geraniol perturbed this activation. These results suggested that suppression of NF-kB might be one of the mechanisms through which geraniol inhibited osteoclast formation.

During osteoclast differentiation RANKL-mediated induction of NFATc1, a master regulator of osteoclastogenesis, is a crucial event that leads to expression of several genes necessary for osteoclast differentiation (Takayanagi 2007). DC-STAMP is highly expressed by differentiating 
osteoclasts where it acts as a regulator of osteoclast cell fusion, but not by precursor macrophages (Yagi et al., 2005). Multinucleated osteoclasts secrete proteolytic enzymes such as CTSK which is directly involved in the breakdown of bone matrix (Delaisse et al., 2003, Ortega et al., 2003). TRAP enzyme dephosphorylates bone sialoprotein and bone matrix phosphoproteins leading to osteoclast migration, and resorption (Ek-Rylander et al., 1994). As a result, we assessed the effects of geraniol on the mRNA expression of NFATc1 and downstream genes. Quantitative RT-PCR analysis showed that geraniol dramatically down-regulated the expression of NFATc1. In addition, other key osteoclast differentiation-related genes were down-regulated, such as DC-STAMP and TRAP possibly due to the inhibition of NFATc1 expression.

\section{Conclusion}

The results of this study provide evidence that geraniol inhibits osteoclastogenesis through the suppression of NF-kB activity, down-regulation of NFATc1 and expression of osteoclastogenic genes in a RANKL-induced osteoclast differentiation model. These findings may suggest a new application of geraniol for enhancing bone health. Further studies using in vivo osteoporotic models will be helpful in establishing the efficacy of geraniol as an anti-osteoclastogenic agent.

\section{Acknowledgments}

This study was supported by grants from the University of Pretoria Vice Chancellor's Postdoctoral Research Fellowship; RESCOM, University of Pretoria and the University of Pretoria's Strategic Institutional Research Theme in Food, Nutrition and Well-being.

\section{Conflict of interest}

The authors declare that they have no conflict of interest. 


\section{Ethical approval}

All procedures performed in studies involving human participants were in accordance with the ethical standards of the Human Research Ethics Committee of the Faculty of Health Sciences, University of Pretoria (Protocol approval number: 67/2014) and with the 1964 Helsinki declaration and its later amendments. Informed consent was obtained from all individual participants included in the study.

\section{References}

Boyle WJ, Simonet WS, Lacey DL (2003) Osteoclast differentiation and activation. Nature 423:337-342

Chen W, Viljoen AM (2010) Geraniol - A review of a commercially important fragrance material. S Afr J Bot 76:643-651

Costa AG, Cusano NE, Silva BC, Cremers S, Bilezikian JP (2011) Cathepsin K: its skeletal actions and role as a therapeutic target in osteoporosis. Nat Rev Rheumatol 7:447-456

Cote GM (2015) Rank ligand as a target in musculoskeletal neoplasms. Curr Rev Musculoskelet Med 8:339-343

Dai S, Hirayama T, Abbas S, Abu-Amer Y (2004) The IkappaB kinase (IKK) inhibitor, NEMObinding domain peptide, blocks osteoclastogenesis and bone erosion in inflammatory arthritis. J Biol Chem 279:37219-37222

Deepak V, Kasonga A, Kruger MC, Coetzee M (2015) Inhibitory effects of eugenol on RANKLinduced osteoclast formation via attenuation of NF-kappaB and MAPK pathways. Connect Tissue Res 56:195-203

Deepak V, Kruger MC, Joubert A, Coetzee M (2015) Piperine alleviates osteoclast formation through the p38/c-Fos/NFATc1 signaling axis. Biofactors 41:403-413

Delaisse JM, Andersen TL, Engsig MT, Henriksen K, Troen T, Blavier L (2003) Matrix metalloproteinases (MMP) and cathepsin K contribute differently to osteoclastic activities. Microsc Res Tech 61:504-513

Dias DA, Urban S, Roessner U (2012) A historical overview of natural products in drug discovery. Metabolites 2:303-336

Drake MT, Clarke BL, Lewiecki EM (2015) The Pathophysiology and Treatment of Osteoporosis. Clin Ther 37:1837-1850 
Ek-Rylander B, Flores M, Wendel M, Heinegard D, Andersson G (1994) Dephosphorylation of osteopontin and bone sialoprotein by osteoclastic tartrate-resistant acid phosphatase. Modulation of osteoclast adhesion in vitro. J Biol Chem 269:14853-14856

Feng R, Anderson G, Xiao G, Elliott G, Leoni L, Mapara MY, Roodman GD, Lentzsch S (2007) SDX-308, a nonsteroidal anti-inflammatory agent, inhibits NF-kappaB activity, resulting in strong inhibition of osteoclast formation/activity and multiple myeloma cell growth. Blood 109:2130-2138

Fonseca D, Ward WE (2004) Daidzein together with high calcium preserve bone mass and biomechanical strength at multiple sites in ovariectomized mice. Bone 35:489-497

Hadjidakis DJ, Androulakis, II (2006) Bone remodeling. Ann N Y Acad Sci 1092:385-396

Hayman AR (2008) Tartrate-resistant acid phosphatase (TRAP) and the osteoclast/immune cell dichotomy. Autoimmunity 41:218-223

Iotsova V, Caamano J, Loy J, Yang Y, Lewin A, Bravo R (1997) Osteopetrosis in mice lacking NF-kappaB1 and NF-kappaB2. Nat Med 3:1285-1289

Kim SH, Bae HC, Park EJ, Lee CR, Kim BJ, Lee S, Park HH, Kim SJ, So I, Kim TW, Jeon JH (2011) Geraniol inhibits prostate cancer growth by targeting cell cycle and apoptosis pathways. Biochem Biophys Res Commun 407:129-134

McGrath EE (2011) OPG/RANKL/RANK pathway as a therapeutic target in cancer. J Thorac Oncol 6:1468-1473

Medicherla K, Sahu BD, Kuncha M, Kumar JM, Sudhakar G, Sistla R (2015) Oral administration of geraniol ameliorates acute experimental murine colitis by inhibiting pro-inflammatory cytokines and NF-kappaB signaling. Food Funct 6:2984-2995

Mundy GR (1999) Bone Remodelling and its Disorders: Taylor \& Francis.

Ortega N, Behonick D, Stickens D, Werb Z (2003) How proteases regulate bone morphogenesis. Ann N Y Acad Sci 995:109-116

Singh B, Sharma RA (2015) Plant terpenes: defense responses, phylogenetic analysis, regulation and clinical applications. 3 Biotech 5:129-151

Soysa NS, Alles N (2009) NF-kappaB functions in osteoclasts. Biochem Biophys Res Commun $378: 1-5$

Strait K, Li Y, Dillehay DL, Weitzmann MN (2008) Suppression of NF-kappaB activation blocks osteoclastic bone resorption during estrogen deficiency. Int J Mol Med 21:521-525 
Takayanagi H (2007) The role of NFAT in osteoclast formation. Ann N Y Acad Sci 1116:227237

Tat SK, Pelletier JP, Velasco CR, Padrines M, Martel-Pelletier J (2009) New perspective in osteoarthritis: the OPG and RANKL system as a potential therapeutic target? Keio J Med $58: 29-40$

Tsai ML, Lin CC, Lin WC, Yang CH (2011) Antimicrobial, antioxidant, and anti-inflammatory activities of essential oils from five selected herbs. Biosci Biotechnol Biochem 75:19771983

Uchiyama S, Yamaguchi M (2005) beta-cryptoxanthin stimulates cell differentiation and mineralization in osteoblastic MC3T3-E1 cells. J Cell Biochem 95:1224-1234

Vaira S, Alhawagri M, Anwisye I, Kitaura H, Faccio R, Novack DV (2008) RelA/p65 promotes osteoclast differentiation by blocking a RANKL-induced apoptotic JNK pathway in mice. J Clin Invest 118:2088-2097

Vaira S, Johnson T, Hirbe AC, Alhawagri M, Anwisye I, Sammut B, O'Neal J, Zou W, Weilbaecher KN, Faccio R, Novack DV (2008) RelB is the NF-kappaB subunit downstream of NIK responsible for osteoclast differentiation. Proc Natl Acad Sci U S A 105:3897-3902

Visagie A, Kasonga A, Deepak V, Moosa S, Marais S, Kruger MC, Coetzee M (2015) Commercial Honeybush (Cyclopia spp.) Tea Extract Inhibits Osteoclast Formation and Bone Resorption in RAW264.7 Murine Macrophages-An in vitro Study. Int J Environ Res Public Health 12:13779-13793

Wada T, Nakashima T, Hiroshi N, Penninger JM (2006) RANKL-RANK signaling in osteoclastogenesis and bone disease. Trends Mol Med 12:17-25

Yagi M, Miyamoto T, Sawatani Y, Iwamoto K, Hosogane N, Fujita N, Morita K, Ninomiya K, Suzuki T, Miyamoto K, Oike Y, Takeya M, Toyama Y, Suda T (2005) DC-STAMP is essential for cell-cell fusion in osteoclasts and foreign body giant cells. J Exp Med 202:345-351

Yamaguchi M, Weitzmann MN (2009) The estrogen 17beta-estradiol and phytoestrogen genistein mediate differential effects on osteoblastic NF-kappaB activity. Int J Mol Med 23:297301 


\section{Figure Legends}

Figure 1. Effects of geraniol on cell viability of CD14+ monocytes and RAW264.7 macrophages. A) CD14+ monocytes, B) RAW264.7 macrophages were treated with indicated concentrations of geraniol for $48 \mathrm{~h}$ and cell viability of proliferating cells was measured using alamar blue assay. The results are mean \pm SD percent of control and are representative of three independent experiments performed in triplicate.

Figure 2. Geraniol inhibits osteoclast formation. A, B) CD14+ monocytes, C, D) RAW264.7 macrophages were differentiated with RANKL alone or in combination with geraniol $(\mathrm{G})$ at indicated concentrations. Osteoclasts stain purple/pink in the presence of TRAP (scale bars: $50 \mu \mathrm{m}$ ). Results are representative of three independent experiments performed in triplicate. $(* * * P<0.001, * P<0.05$ vs. RANKL).

Figure 3. Geraniol inhibits actin ring formation and bone resorption. RAW264.7 macrophages were differentiated into osteoclasts in the presence of RANKL alone or with geraniol $(23 \mu \mathrm{g} / \mathrm{ml})$ and were stained for A) actin ring formation with phalloidin (Scale bars: $20 \mu \mathrm{m}$ ). or B) were tested for resorption pit formation on osteoassay plates (Scale bars: $50 \mu \mathrm{m}$ ). Resorption percentages quantified with ImageJ are indicated in the figure.

Figure 4. Geraniol suppresses RANKL-induced NF-kB activation. RAW264.7 macrophages stably transfected with NF-kB-pNIFTY2-SEAP plasmids were either treated with RANKL alone or in combination with indicated concentrations of geraniol. SEAP activity from culture supernatants were measured after $48 \mathrm{~h}$. Data shown is SEAP activity \pm SD percent of control. Results are representative of three independent experiments performed in triplicate. (*** $P<0.001$, $* * P<0.01$ vs. RANKL).

Figure 5. Geraniol downregulates osteoclast specific gene expression. RAW264.7 macrophages were treated with RANKL in the presence or absence of geraniol $(23 \mu \mathrm{g} / \mathrm{ml})$ as mentioned in methods for 5 days. qRT-PCR was performed to analyze the expression of CTSK, TRAP, DC-STAMP and NFATc1. Data are expressed as mean $\pm \mathrm{SD}$ and are representative of three independent experiments. ( $* * * P<0.01 \mathrm{vs.} \mathrm{RANKL).}$ 
Figure 1
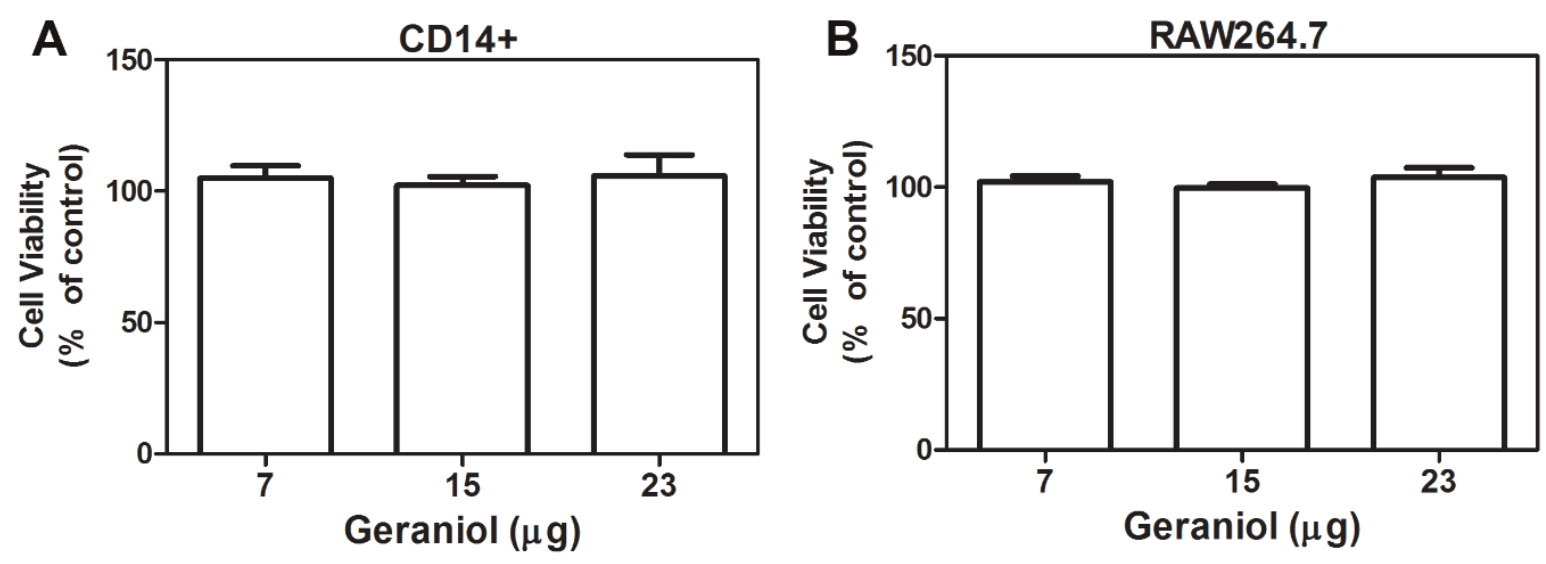
Figure 2

A
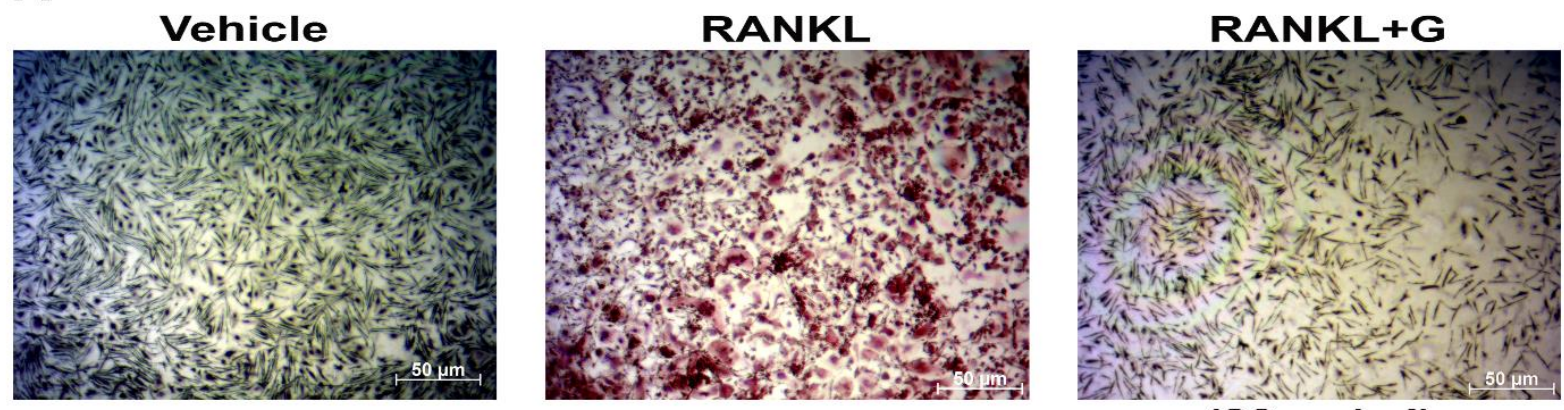

(23 $\mathrm{\mu g} / \mathrm{ml}$ )

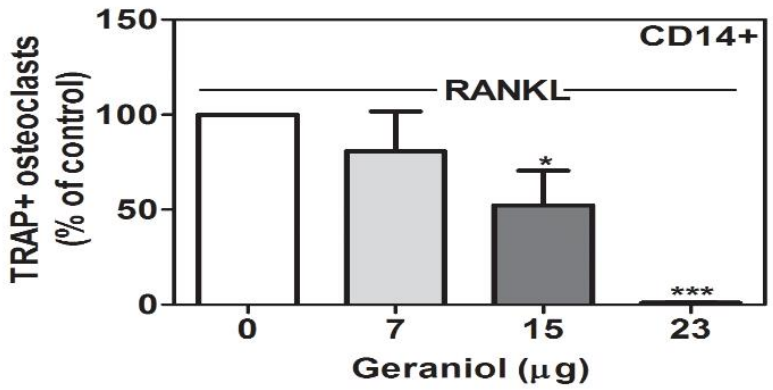

B
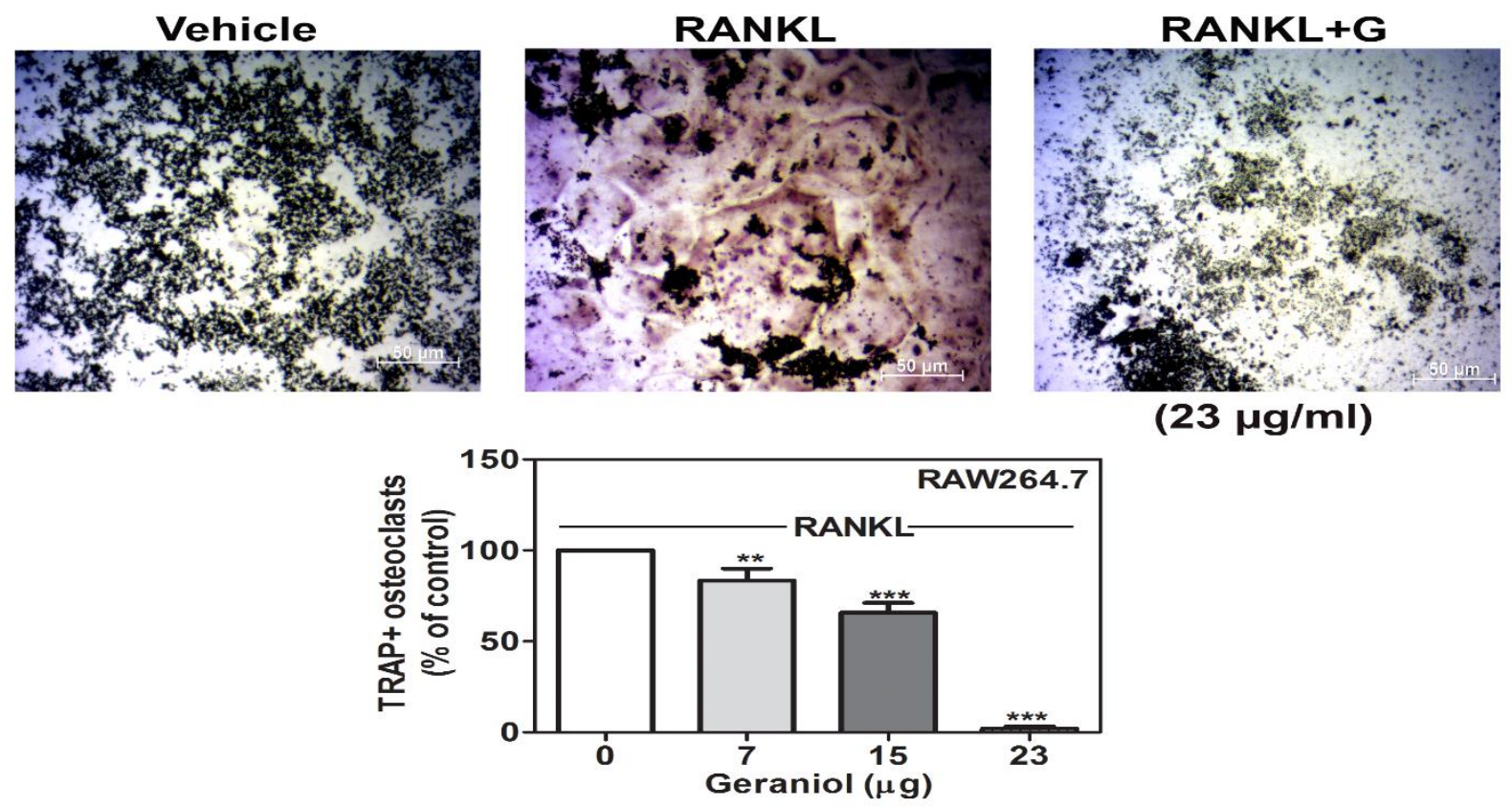

(23 $\mathrm{\mu g} / \mathrm{ml}$ ) 
Figure 3
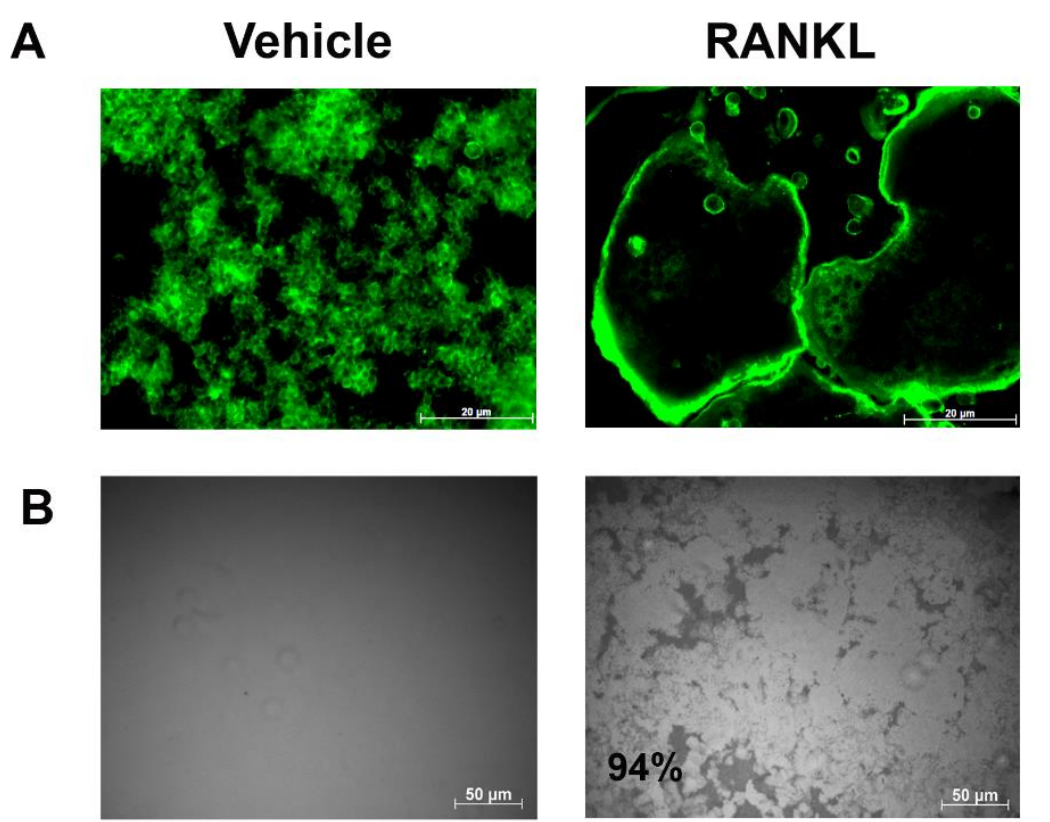

RANKL+G $(23 \mu \mathrm{g} / \mathrm{ml})$
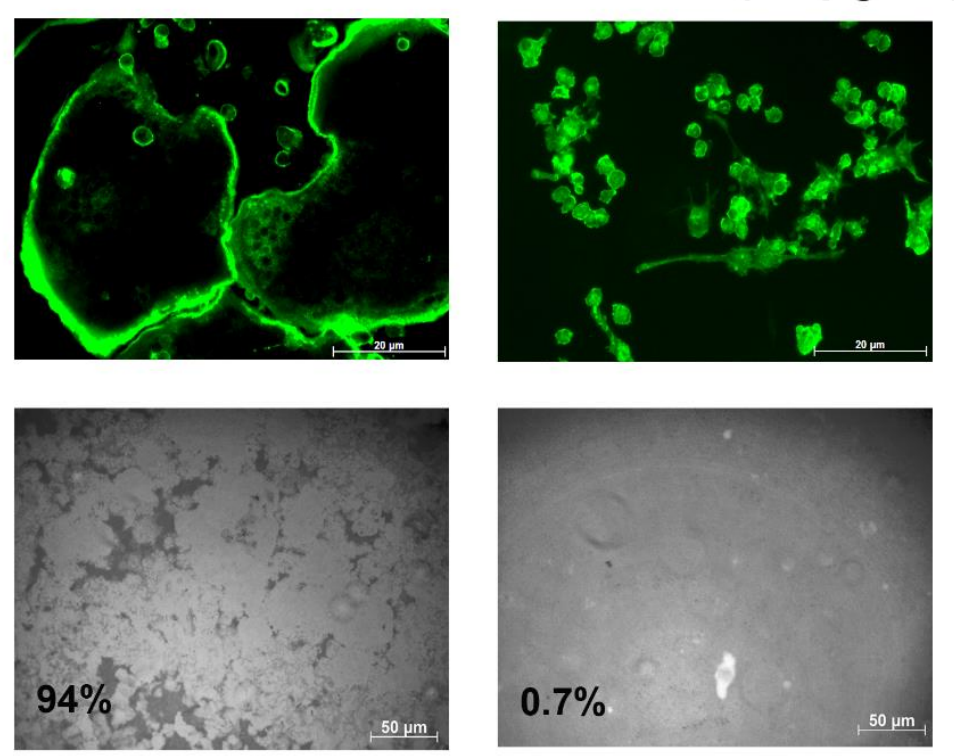
Figure 4

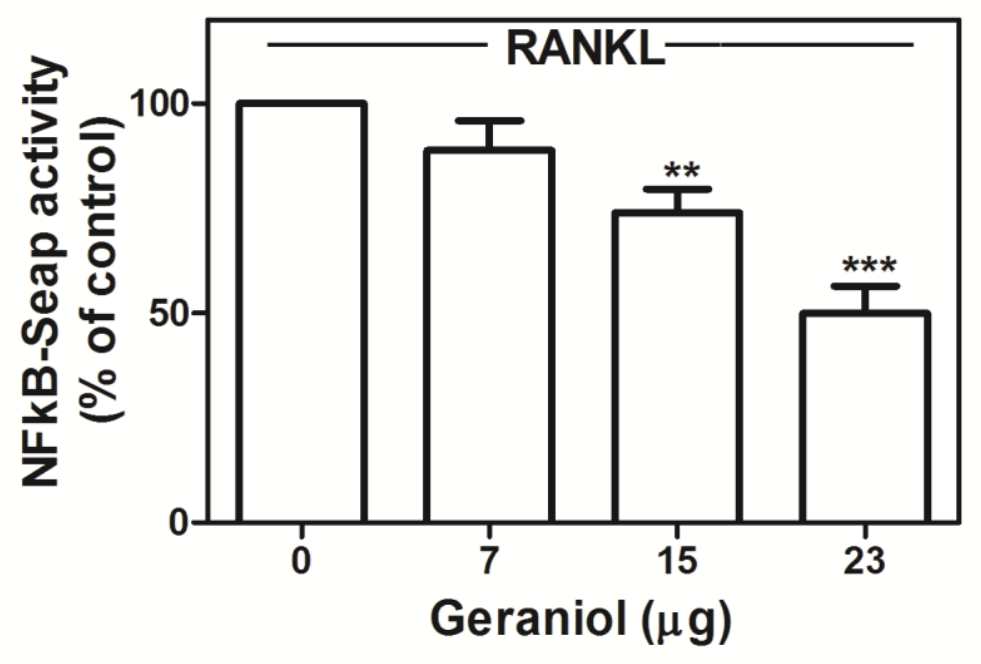


Figure 5
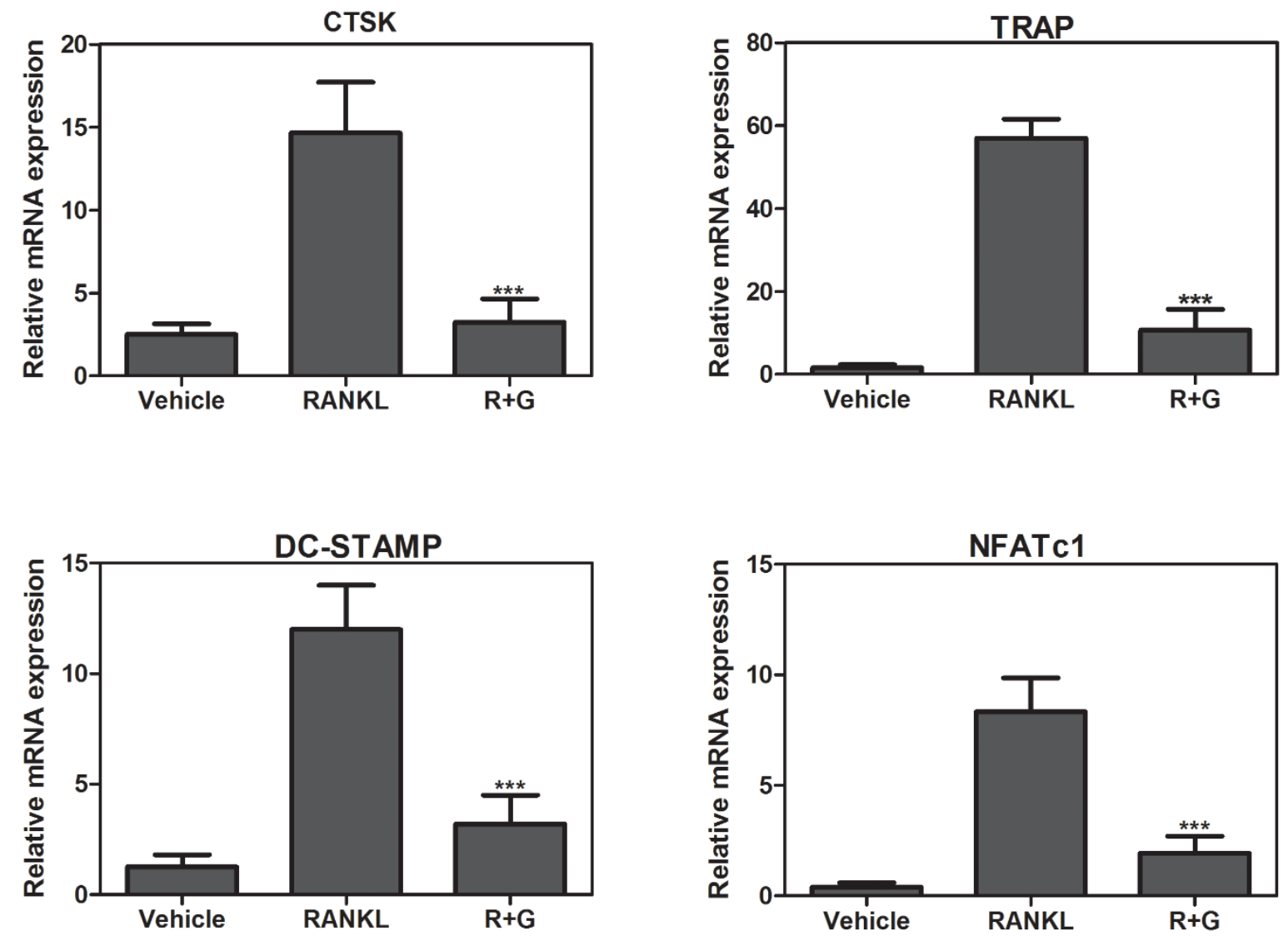